\title{
Intravitreal Clindamycin in the Treatment of Unresponsive Zone One Toxoplasmic Chorioretinitis: A Case Report
}

\author{
Seyedeh Maryam Hosseini ${ }^{1}$; Mojtaba Abrishami ${ }^{1,2,{ }^{*}}$; Mehran Mehdi Zadeh $^{1}$

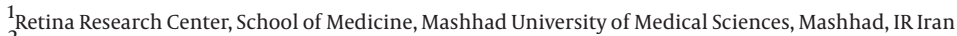 \\ ${ }^{2}$ Eye Research Center, Farabi Eye Hospital, Tehran University of Medical Sciences, Tehran, IR Iran \\ ${ }^{*}$ Corresponding Author: Mojtaba Abrishami, Eye Research Center, Farabi Eye Hospital, Tehran University of Medical Sciences, Tehran, IR Iran. Tel: +98-2155400003, E-mail: mojtaba_ \\ abrishami@yahoo.com
}

Received: October 15, 2013; Revised: August 30, 2014; Accepted: September 2, 2014

\begin{abstract}
Introduction: The current study aimed to report a case of toxoplasma chorioretinitis resistant to standard treatments that dramatically responded to intravitreal clindamycin injection.

Case Presentation: A 23-year-old woman with the diagnosis of ocular toxoplasmosis in the left eye was initially treated by oral pyrimethamine, sulfadiazine, azithromycin and oral prednisolone. Since the treatment was unsuccessful intravitreal clindamycin (1 mg/ $0.1 \mathrm{~mL}$ ) was injected. Responding dramatically, visual acuity improved from hand motion to 20/60 and 20/20, after seven days and six weeks, respectively. Anterior chamber and vitreous reactions were resolved.

Conclusions: Intravitreal injection of clindamycin may be an acceptable alternative to the classic treatment in cases with refractory ocular toxoplasmosis. It may offer the patient more convenience, a safer systemic side effect profile, greater availability, and fewer followup visits and hematologic evaluations. Moreover, since it responds dramatically, it would be helpful in cases with involvement of macula or closeness to the optic nerve.
\end{abstract}

Keywords:Chorioretinitis; Clindamycin; Intraocular Injection

\section{Introduction}

Toxoplasma gondii is an intracellular parasite which is the most common cause of posterior uveitis in the world (1). Although it is usually a self-limited disease, it may reduce vision secondary to optic nerve or macular involvement and/or severe vitreous inflammation. Generally, indications for systemic treatment of ocular toxoplasmosis include location of the active lesion within zone one of the retina, a lesion greater than two disc diameters with $3+$ or more vitreous cells, and any active toxoplasmic lesion in the immunocompromised patients (2). Treatment aims to reduce the parasite multiplication during the active period of chorioretinitis (3). The traditional medications include sulfadiazine, pyrimethamine with folinic acid, clindamycin, and trimethoprim-sulfamethoxazole (TMP-SMZ) double strength. The synergistic combination of pyrimethamine and sulfadiazine is the most common therapy used $(4,5)$. In a randomized trial TMP-SMZ was compared with pyrimethamine and sulfadiazine and the results showed similar efficacy of the two treatment regimens in the ocular toxoplasmosis (6). More recently, other antimicrobials such as azithromycin and atovaquone were used successfully. However, some patients are intolerant , allergic, or infection resistant to the systemic therapy (7). By bypassing ocular barriers and intravitreal antibiotics injection can deliver a high concentration of drug to the intraocular tissues. Recent reports showed the efficiency of intravitreal clindamycin with or without dexamethasone in the treatment of toxoplasmic chorioretinitis (8-11). The current study reported a patient resistant to the standard treatment of toxoplasma retinitis that was successfully managed by intravitreal clindamycin injection.

\section{Case Presentation}

A 23-year-old woman with the chief complaint of reduced vision of the left eye for a week was referred to the emergency department of Khatam-al-Anbia Ophthalmology Clinic, Mashhad, Iran on January 2013. In her left eye, the visual acuity (VA) was hand motion (HM). Relative afferent pupillary defect was negative. Slit lamp examination revealed diffuse fine KPs, 2+ anterior chamber reaction, and severe vitritis in the left eye. From a hazy media, active white creamy chorioretinitis at the margin of an old chorioretinal scar was observed. In the right eye, old chorioretinal scar was observed (Figure 1). Due to bilateral chorioretinal scar, and an active site at the margin of an old scar in the involved eye, by the diagnosis of ocular toxoplasmosis in the left eye, she was initially treated by oral azithromycin (500 mg daily) and pyrimethamine (loading dose of $75 \mathrm{mg}$ and then $25 \mathrm{mg}$ daily). After 48 hours, oral prednisolone $(75 \mathrm{mg} /$ day as $1 \mathrm{mg} / \mathrm{kg}$ body

Copyright ( ) 2014, Iranian Red Crescent Medical Journal. This is an open-access article distributed under the terms of the Creative Commons Attribution-NonCommercial 4.0 International License (http://creativecommons.org/licenses/by-nc/4.0/) which permits copy and redistribute the material just in noncommercial usages, provided the original work is properly cited. 
weight) was initiated for the patient. After one week, there was no change in the vision or the ocular inflammation. The laboratory tests disclosed toxoplasmosis IgG sero-positivity not toxo-IgM. As the primary treatment was unsuccessful, oral sulfdiazine (4 gram stat as a loading dose and $500 \mathrm{mg}$ q6h as maintenance) was added for another week. A partial response, and mild decrease in vitritis was achieved one week later but VA was HM. Then, to avoid the involvement of optic disc, intravitreal clindamycin $(1 \mathrm{mg} / 0.1 \mathrm{~mL})$ was injected. The patient responded to the treatment dramatically, (Figure 2) the VA improved from HM to 20/60 and 20/20 after seven days, and six weeks respectively. Anterior chamber and vitreous reactions complications were resolved.

\section{Discussion}

The management of ocular toxoplasmosis in the patients who are unresponsive or intolerant of oral therapy is challenging. Intraocular drug delivery is an option for these patients (10). The indications for intraocular therapy in toxoplasmic chorioretinitis include contraindication to oral medications in pregnancy, disease progression despite oral antimicrobial treatment, lesions located at or near the optic disc or fovea, and intolerance to oral medications $(9,11)$. The patient described herein was a case of unresponsive to the standard oral treatments, but responded dramatically to a single intravitreal injection of clindamycin after one week. Since the need, type, and duration of the treatment was individualized, the patient was initially treated with more synergistic medications. Because of unavailability of sulfadiazine at that time, azithromycin and pyrimethamine were started, but no response was observed. The addition of sulfadiazine was also ineffective. Since the patient had a toxoplasmic chorioretinitis in close proximity to the optic nerve that was unresponsive to the standard oral therapy, after two weeks, to decrease more detrimental effect on vision,

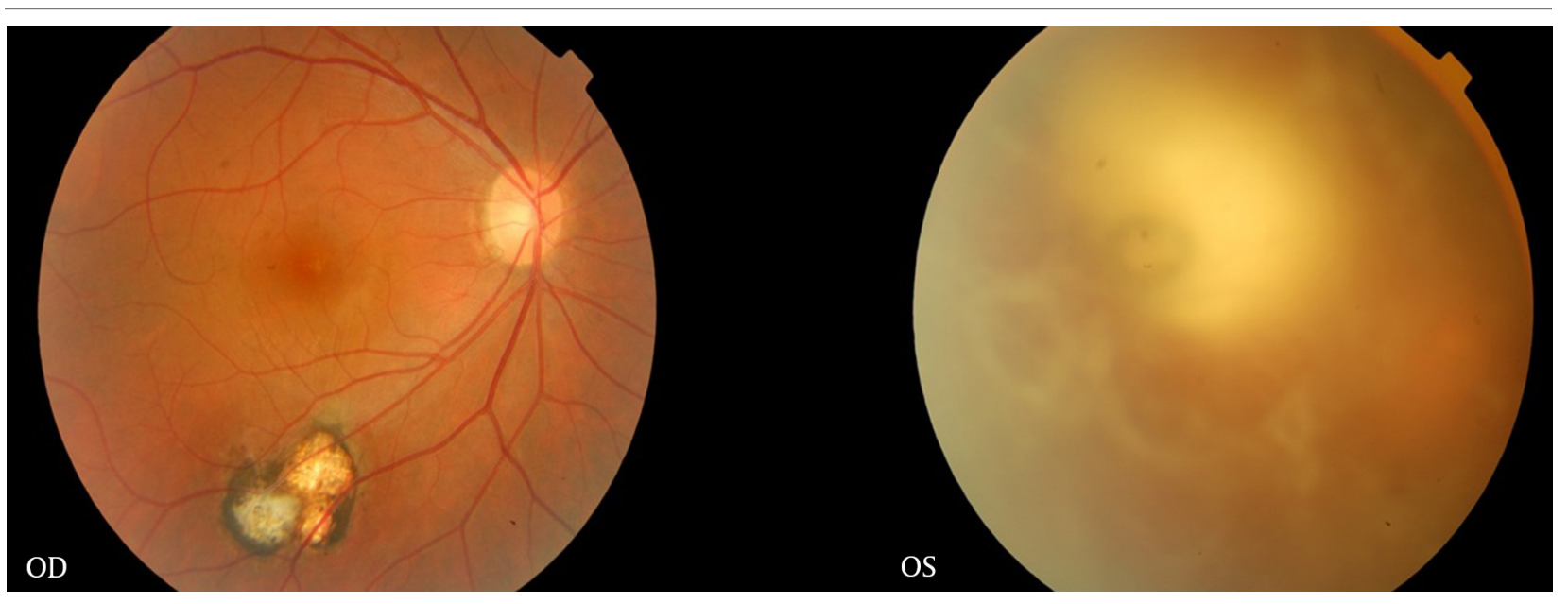

Figure 1. Right and the Left Eyes Before the Treatment

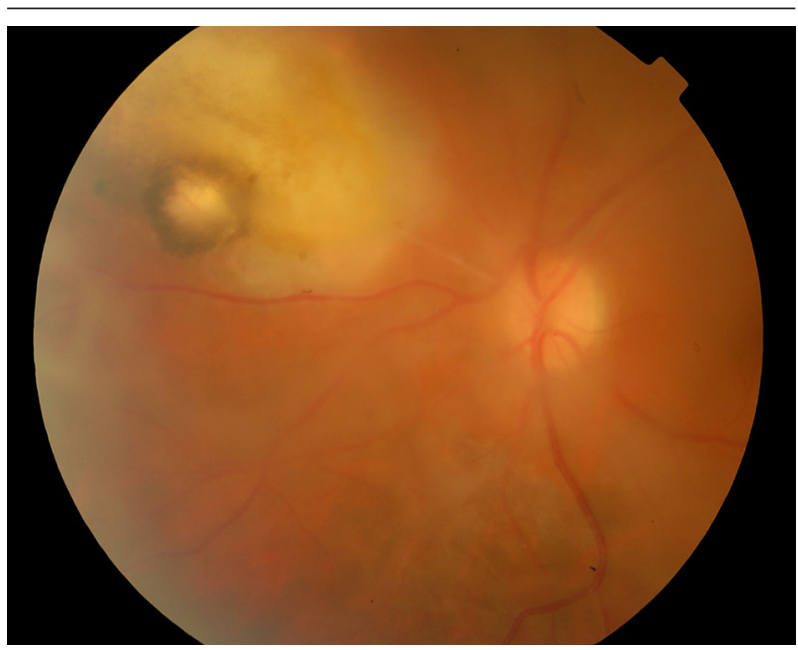

Figure 2. The Left Eye After the Treatment and Intravitreal Clindamycin Injection the intravitreal injection of $1 \mathrm{mg} / 0.1 \mathrm{~mL}$ clindamycin was considered.Sobrin etal. reported sixcases of toxoplasmic chorioretinitis treated with intravitreal clindamycin $1 \mathrm{mg} / 0.1 \mathrm{~mL}$. Indications for the local therapy included intolerance to the systemic medications or disease progression despite systemic antimicrobial treatments. In their series, treatment with single injection was associated with resolution of vitreous inflammation within six weeks in five of six patients (9). Wong et al. reported a toxoplasmic chorioretinitis case with atypical presentation of disc swelling and hemispherical retinal vein occlusion successfully managed by two injections of intravitreal clindamycin and oral steroid because of intolerance to oral treatment (10). Lasave et al. (11) reported functional and anatomical improvement in 12 patients (eyes) with posterior pole or zone one toxoplasmic chorioretinitis treated weekly or every four weeks (in pregnancy) with intravitreal clindamycin $(1.5 \mathrm{mg} / 0.1 \mathrm{~mL})$ and dexamethasone $(0.4 \mathrm{mg} / 0.1 \mathrm{~mL})$. 
Patients were controlled for 24 months. Indications of local therapy included intolerance to oral treatment, contraindication to systemic treatment because of pregnancy, lack of response despite systemic therapy, or combined oral and local treatment in sight-threatening cases to avoid or limit foveal or optic disc involvement (11). Martinez et al. reported successful treatment of sight-threatening toxoplasmic chorioretinitis with intravitreal injection of clindamycin and dexamethasone combined with systemic sulfadiazine in a woman during the first trimester of pregnancy (12). Intravitreal steroid was not used in the current case. The use of intravitreal steroids in TRC is still controversial. Disease reactivation is reported with both intravitreal and periocular steroid treatment (13-15). The patient in the current study received oral medication for four weeks after injection, since prolonged systemic therapy may decrease the need of reinjection (9). This case reemphasizes the effectiveness of intravitreal clindamycin in the treatment of unresponsive toxoplasmic chorioretinitis and sight-threatening cases with zone one toxoplasmic chorioretinitis. Making a clinical trial to evaluate the treatment of toxoplasmosis is needed and case reports or series like the current one are not sufficient to change the treatment protocol. However, patients must be informed about potential risks of the procedure, including endophthalmitis, cataract, and retinal detachment.

\section{Acknowledgements}

The authors would like to thank Maryam Kadkhoda for her help in optometric tests and taking fundus photos of the patient.

\section{Authors' Contributions}

Seyyed Maryam Hoseini developed the original idea, revised the manuscript, supervised the treatment and is guarantor. Mojtaba Abrishami prepared the manuscript, helped in follow ups of the patient and acquisition of data, and finally revised the manuscript. Mehran Mehdo
Zadeh helped in follow ups of the patient and acquisition of data and abstracted the findings.

\section{References}

1. Dodds EM. Ocular toxoplasmosis: clinical presentations, diagnosis and therapy. Focal Points: Clinical Modules for Ophthalmologists.San Francisco: American Academy of Ophthalmology; 1999.

2. Holland GN, Lewis KG. An update on current practices in the management of ocular toxoplasmosis. Am J Ophthalmol. 2002;134(1):102-14.

3. Rothova A, Meenken C, Buitenhuis HJ, Brinkman CJ, Baarsma GS, Boen-Tan TN, et al. Therapy for Ocular Toxoplasmosis. Am J Ophthalmol.1993;115(4):517-23.

4. Engstrom RE, Holland GN, Nussenblatt RB, Jabs DA. Current Practices in the Management of Ocular Toxoplasmosis. Am J Ophthalmol.1991;111(5):601-10.

5. Opremcak E, Scales DK, Sharpe MR. Trimethoprim-Sulfamethoxazole Therapy for Ocular Toxoplasmosis. Ophthalmology. 1992;99(6):920-5.

6. Soheilian M, Sadoughi MM, Ghajarnia M, Dehghan MH, Yazdani S, Behboudi H, et al. Prospective randomized trial of trimethoprim/sulfamethoxazole versus pyrimethamine and sulfadiazine in the treatment of ocular toxoplasmosis. Ophthalmology. 2005;112(11):1876-82.

7. Bonfioli AA, Orefice F. Toxoplasmosis. Semin Ophthalmol. 2005;20(3):129-41.

8. Kishore K, Conway MD, Peyman GA. Intravitreal clindamycin and dexamethasone for toxoplasmic retinochoroiditis. Ophthalmic Surg Lasers. 2001;32(3):183-92.

9. Sobrin L, Kump LI, Foster CS. Intravitreal clindamycin for toxoplasmic retinochoroiditis. Retina. 2007;27(7):952-7.

10. Wong R, dell'Omo R, Marino M, Hussein B, Okhravi N, Pavesio CE. Toxoplasma gondii: an atypical presentation of toxoplasma as optic disc swelling and hemispherical retinal vein occlusion treated with intravitreal clindamycin. Int Ophthalmol. 2009;29(3):195-8.

11. Lasave AF, Diaz-Llopis M, Muccioli C, Belfort RJ, Arevalo JF. Intravitreal clindamycin and dexamethasone for zone 1 toxoplasmic retinochoroiditis at twenty-four months. Ophthalmology. 2010;117(9):1831-8.

12. Martinez CE, Zhang D, Conway MD, Peyman GA. Successful management of ocular toxoplasmosis during pregnancy using combined intraocular clindamycin and dexamethasone with systemic sulfadiazine. Int Ophthalmol. 1998;22(2):85-8.

13. Nicholson DH, Wolchok EB. Ocular Toxoplasmosis in an Adult Receiving Long-Term Corticosteroid Therapy. Archives of Ophthalmology. 1976;94(2):248-54.

14. Nozik RA. Results of treatment of ocular toxoplasmosis with injectable corticosteroids. Trans Sect Ophthalmol Am Acad Ophthalmol Otolaryngol.1977;83(5):811-8.

15. O'Connor GR, Frenkel JK. Editorial: Dangers of steroid treatment in toxoplasmosis. Periocular injections and systemic therapy. Arch Ophthalmol.1976;94(2):213. 